\title{
A Bioinformatics Investigation into the Pharmacological Mechanisms of Sodium-Glucose Co-transporter 2 Inhibitors in Diabetes Mellitus and Heart Failure Based on Network Pharmacology
}

\author{
Ziling Mai ${ }^{1,2} \cdot$ Huanqiang $\mathrm{Li}^{2} \cdot$ Guanzhong Chen ${ }^{2,3} \cdot$ Enzhao Chen ${ }^{2} \cdot$ Liwei Liu $^{2,4} \cdot$ Zhubin Lun $^{2,5} \cdot$ Wenguang Lai ${ }^{1,2}$.

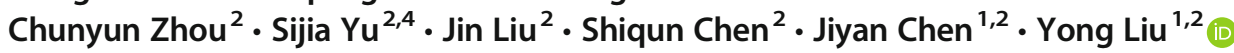

Accepted: 5 April 2021 / Published online: 24 May 2021

(C) The Author(s) 2021

\begin{abstract}
Purpose Diabetes mellitus (DM) is a major risk factor for the development of heart failure (HF). Sodium-glucose co-transporter 2 (SGLT2) inhibitors have demonstrated consistent benefits in the reduction of hospitalization for HF in patients with DM. However, the pharmacological mechanism is not clear. To investigate the mechanisms of SGLT2 inhibitors in DM with HF, we performed target prediction and network analysis by a network pharmacology method.

Methods We selected targets of SGLT2 inhibitors and DM status with HF from databases and studies. The "Drug-Target" and "Drug-Target-Disease" networks were constructed using Cytoscape. Then the protein-protein interaction (PPI) was analyzed using the STRING database. Gene Ontology (GO) biological functions and Kyoto Encyclopedia of Genes and Genomes (KEGG) pathways were performed to investigate using the Bioconductor tool for analysis.

Results There were 125 effective targets between SGLT2 inhibitors and DM status with HF. Through further screening, 33 core targets were obtained, including SRC, MAPK1, NARS, MAPK3 and EGFR. It was predicted that the Rap1 signaling pathway, MAPK signaling pathway, EGFR tyrosine kinase inhibitor resistance, AGE-RAGE signaling pathway in diabetic complications and other signaling pathways were involved in the treatment of DM with HF by SGLT2 inhibitors.

Conclusion Our study elucidated the possible mechanisms of SGLT2 inhibitors from a systemic and holistic perspective based on pharmacological networks. The key targets and pathways will provide new insights for further research on the pharmacological mechanism of SGLT2 inhibitors in the treatment of DM with HF.
\end{abstract}

Keywords Diabetes mellitus $\cdot$ Heart failure $\cdot$ Sodium-glucose co-transporter 2 inhibitors $\cdot$ Network pharmacology

\section{Introduction}

Diabetes mellitus $(\mathrm{DM})$ is a metabolic disorder characterized by hyperglycemia due to partial or complete insulin deficiency

Ziling Mai, Huanqiang Li, Guanzhong Chen, Enzhao Chen and Liwei Liu contributed equally to this work.

Jiyan Chen

chenjiyandr@126.com

$\triangle$ Yong Liu

liuyong@gdph.org.cn

1 Guangdong Provincial People's Hospital, School of Biology and Biological Engineering, South China University of Technology, Guangzhou, China and/or insulin resistance, which affects $8.5-9.3 \%$ of the population worldwide, rising to an expected $10.2 \%$ (578 million) by 2030 and $10.9 \%$ (700 million) by $2045[1,2]$. Heart failure (HF) is a substantial but frequently overlooked complication

2 Department of Cardiology, Guangdong Provincial Key Laboratory of Coronary Heart Disease Prevention, Guangdong Cardiovascular Institute, Guangdong Provincial People's Hospital, Guangdong Academy of Medical Sciences, Guangzhou, China

3 Guangdong Provincial People's Hospital, School of Medicine, South China University of Technology, Guangzhou, China

4 The Second School of Clinical Medicine, Southern Medical University, Guangzhou, China

5 The First School of Clinical Medicine, Guangdong Medical University, Zhanjiang, China 
of DM [3]. Several studies show that the incidence of HF is 25 times as high in diabetic patients as in those without DM [4]. Furthermore, diabetic patients with HF have longer HFrelated hospital stays, more frequent HF-related readmissions and higher risk for cardiovascular mortality than patients with HF but without DM [5-7]. However, treating patients with these concomitant diseases can be challenging. Some drugs have been recommended for the treatment of DM with HF, such as metformin and sulfonylureas, but they are insufficient in treating diabetes with heart failure. For instance, metformin alone is often not enough to keep glycemia under control, and thus does not significantly improve patients' condition [8]. Sulfonylureas, used as second-line or third-line treatments for diabetic patients with heart failure according to the position statement of the European Society of Cardiology/Heart Failure Association, are commonly prescribed in DM but associated with weight gain and hypoglycemia, which are detrimental in heart failure [9-11]. Therefore, there is a compelling impetus to explore potential strategies to reduce the risk of $\mathrm{HF}$ in patients with DM.

Sodium-glucose co-transporter 2 (SGLT2) inhibitors, a class of glucose-lowering therapies, including dapagliflozin, canagliflozin, empagliflozin and ertugliflozin, have been approved by the US Food and Drug Administration for the treatment of type 2 diabetes mellitus [12]. SGLT2 inhibitors can inhibit proximal renal tubular sodium and glucose reabsorption to increase the urinary excretion of glucose, thereby reducing blood sugar [13]. Data to date suggest that this agent achieves a statistically significant and clinically relevant reduction in the risk of $\mathrm{CV}$, renal outcomes and overall mortality beyond simply reducing plasma glucose levels. Clinical trials from many countries, including the EASEL Population-Based Cohort Study [14], the EMPA-REG OUTCOME trial [15] and the CVD-REAL 2 study [16], have proven that SGLT2 inhibitors reduce the risk of nonfatal myocardial infarction, non-fatal stroke, and all-cause death, as well as the risk of hospitalization and death associated with heart failure. Moreover, it can reduce the decline in renal function to protect patients from dialysis and renal failure as reported in the DARWIN-T2D and a Scandinavian cohort study $[17,18]$. Most attention has focused on the pleiotropic effects of SGLT2 inhibitors on cardiac function and their potential benefits with regard to heart failure and mortality rates. Four large cardiovascular outcomes trials, the EMPAREG OUTCOME trial [15], the CANVAS Program 30 [19], the DECLARETIMI 58 trial [20] and the VERTIS CV [21] trial, have demonstrated a significant and consistent reduction in heart failure events with SGLT2 inhibitors. Based on these composite data, SGLT2 inhibitors represent an important new therapeutic approach for the prevention of heart failure in at-risk patients with diabetes mellitus.
And this benefit extends to patients without diabetes who have heart failure with reduced ejection fraction. The DAPA-HF study is a prospective randomized placebo-controlled trial to determine the effectiveness and safety of dapagliflozin in patients with heart failure with reduced ejection fraction based on conventional treatment. The results of the trial showed that, compared with placebo, patients with DM experienced a similar reduction in the risk of hospitalization for HF as patients without DM. This finding suggests that other actions of SGLT2 inhibitors beyond glucose-lowering might play a role in the beneficial effects of these agents in patients with HF. Therefore, the results of this large study further support the benefits of SGLT2 inhibitors in the treatment of HF [22]. However, the therapeutic targets and mechanism of SGLT2 inhibitors acting on HF have not yet been revealed, especially in patients with DM, which needs to be further explored and analyzed.

With the development of high-throughput sequencing and computer technology, huge new bioinformatics networks have emerged. Network pharmacology, one such bioinformatics network, aims to construct a multilevel network through various database searches, highthroughput omics data analysis and computer simulations to analyze the relationship of medicines, diseases and targets [23]. Compared with traditional experimental pharmacology methods, network pharmacology is based on a comprehensive system which is more efficient for exploring the target and pathway relationships between drugs and diseases. Therefore, we applied network pharmacology analysis to systematically excavate the action targets of SGLT2 inhibitors in DM with HF to analyze the biological pathways involved, which will lay a good foundation for further in-depth exploration of the mechanism of SGLT2 inhibitors acting on DM with HF. The study flowchart of this network pharmacology-based study of SGLT2 inhibitors is shown in Fig. 1.

\section{Methods}

\section{Prediction of SGLT2 Inhibitor-Related Targets}

The chemical structures of four SGLT2 inhibitors, namely canagliflozin, dapagliflozin, empagliflozin, and ertugliflozin, were obtained using Pubchem (https://pubchem.ncbi.nlm.nih. gov/), which is an open chemistry database with 96,502,248 compositions, of which $3,151,393$ have been tested. Then SwissTargetPrediction (http://www.swisstargetprediction. $\mathrm{ch} /$ ), a tool for target prediction according to twodimensional and three-dimensional similarity measures with known ligands, was selected to predict potential targets for four SGLT2 inhibitors by putting their chemical structures 
Fig. 1 The workflow of the network pharmacology strategies for determining the pharmacological mechanisms of the SGLT2 inhibitors in diabetes with heart failure through cluster and pathway analysis.

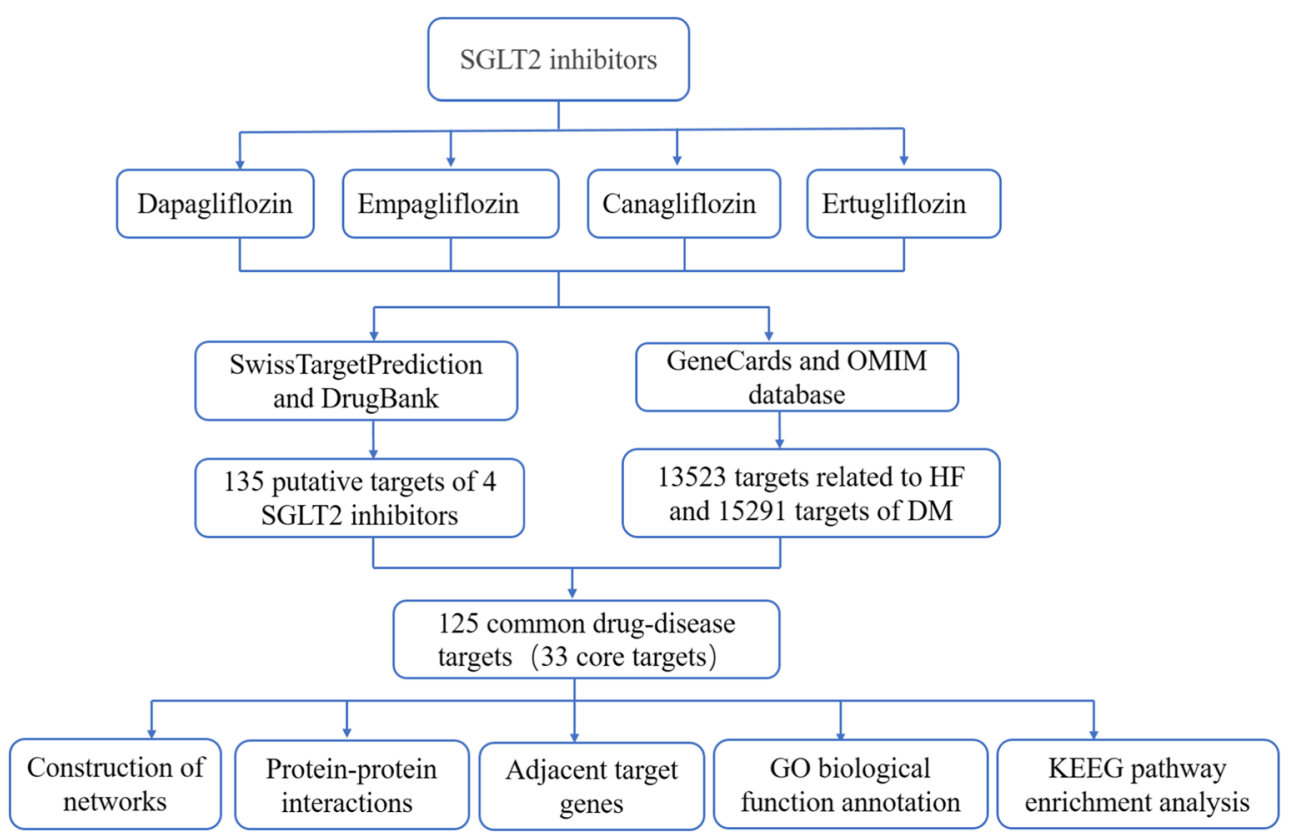

into this platform [24]. Additionally, SGLT2 inhibitor-related genes were collected from DrugBank (https://www.drugbank. $\mathrm{ca} /$ ), which is a unique bioinformatics and chemical informatics database, containing 11,628 drugs and related chemical information, drug targets, protein data, and so on [25]. With further correction and transformation by the retrieval of Universal Protein Resource (UniProt, http:// www.uniprot.org/), all the SGLT2 inhibitor-related genes were normalized into consistent symbols for subsequent analysis.

\section{The Prediction of Known Therapeutic Targets in DM Status with HF}

With the keywords of "heart failure", "diabetes", and "diabetes mellitus", target genes related to HF and DM were found in the GeneCards (https://www.genecards.org) and OMIM (https://www.omim.org) databases. The GeneCards database includes more than 7000 human genes, and each gene has an approved gene symbol. The OMIM database is a knowledge base of human genes and hereditary diseases. The two methods are a good reference for the collection of disease targets. Meanwhile, we collected disease targets from the studies reporting existing network analysis on actual patients with DM status with HF [26, 27]. According to the targets of SGLT2 inhibitors and diseases, the repeated targets of the two were screened by Excel, and their intersection targets were obtained. According to their intersection, we obtained a Venn diagram on the Venny website (https://bioinfogp.cnb.csic.es/tools/venny/).

\section{Construction of the Network Model}

The four SGLT2 inhibitors, their corresponding targets and the intersection targets of diseases and drugs were sorted and input into Cytoscape v3.6.1 to construct the following networks: (1) network between four SGLT2 inhibitors (canagliflozin, dapagliflozin, empagliflozin, and ertugliflozin) and their corresponding targets; (2) network between four SGLT2 inhibitors, intersection targets, and diseases (DM status with HF). Cytoscape is a software program that can efficiently express the interaction between protein and protein, protein and DNA or gene, in order to visualize network relationships.

\section{Screening of Core Targets of SGLT2 Inhibitors in Treatment of DM with HF}

The STRING (https://string-db.org) dataset is now one of the largest PPI datasets, including co-expression data, biomedical literature data, high-throughput data and genomic background data. In the platform, "Multiple proteins" was selected and the organism was selected as "Homo sapiens. "The intersection targets of SGLT2 inhibitors and DM status with HF were then imported to construct the protein-protein interaction network. In order to ensure the high confidence of information, the scoring condition was set to $>0.90$, and the isolated proteins in the figure were hidden. The file was exported in "TSV" format, and then Cytoscape v3.6.1 was used to analyze the topological properties of the PPI network. To detect the core targets in the common targets between drugs and diseases, the 
degrees of targets were calculated using the Cytohubba plugin based on Cytoscape.

\section{Construction of Protein-Protein Interaction (PPI) Network of Core Targets}

The STRING online platform was used to build the PPI network of core targets. After selecting the multiple proteins module, the Gene Name list of target proteins was uploaded to the network, limiting the species to Homo sapiens, and the confidence score was set to $>0.9$. In the PPI diagram, each solid circle represents a gene, and the middle of the circle shows the structure of the protein, while the circles are connected by lines of different colors. Each line represents the biological process between protein and protein, including regulation of gene expression, signal transduction, cell migration and so on.

\section{GO Enrichment Analysis and KEGG Pathway Enrichment Analysis}

In order to better understand the potential biological process of core genes, KEGG (Kyoto Encyclopedia of Genes and Genomes) and GO (Gene Ontology) were analyzed for pathway functional enrichment using the clusterProfiler software package on the $\mathrm{R}$ platform. The interaction network was constructed using the Top-Go package of the R platform.

\section{Results}

\section{Screening Potential Related Targets of SGLT2 Inhibitors}

The PubChem platform was used to acquire the molecular structure of dapagliflozin empagliflozin, canagliflozin and ertugliflozin. The details of their structure are shown in Table 1. According to their molecular structure, the four SGLT2 inhibitors have a common parent nucleus, although their side-chain substituents are somewhat different. After importing their structures into the SwissTargetPrediction database for target matching and prediction, we screened the 213 targets with probability $>0$, of which 71 targets were in dapagliflozin, 65 in canagliflozin, 61 in ertugliflozin and 16 in empagliflozin. We also accessed the DrugBank database to find 37 targets on the four SGLT2 inhibitors, with 11 targets in dapagliflozin, 10 in empagliflozin, 8 in canagliflozin and 8 in ertugliflozin. By integrating and eliminating duplicate targets in the two databases, a total of 135 targets with potential effects of SGLT2 inhibitors were obtained. Then, introducing them into Cytoscape v3.6.1 software for analysis, we

Table 1 Information on four SGLT2 inhibitors from PubChem

\begin{tabular}{lllll}
\hline SGLT2 inhibitors & $\begin{array}{l}\text { PubChem } \\
\text { CID }\end{array}$ & $\begin{array}{l}\text { Molecular } \\
\text { Formula }\end{array}$ & 2D Image & 3D Image \\
\hline Dapagliflozin & 9887712 & $\mathrm{C}_{21} \mathrm{H}_{25} \mathrm{ClO}_{6}$ & & \\
\hline Canagliflozin & 24812758 & $\mathrm{C}_{24} \mathrm{H}_{25} \mathrm{FO}_{5} \mathrm{~S}$ & & \\
\hline Empagliflozin & 11949646 & $\mathrm{C}_{23} \mathrm{H}_{27} \mathrm{ClO}_{7}$ & \\
\hline Ertugliflozin & 44814423 & $\mathrm{C}_{22} \mathrm{H}_{25} \mathrm{ClO}_{7}$ & \\
\hline
\end{tabular}


constructed a visualized drug-target network (Fig. 2). There are 139 nodes (135 targets, 4 drugs) and 252 edges shown in Fig. 2, where the pink nodes represent SGLT2 inhibitors, the blue nodes represent the drug targets (the predicted target) and the edges represent the interactions between the drugs and the targets. This reflects the potential mechanism of interaction between SGLT2 inhibitors and multiple targets.

\section{Construction and Analysis of Drug-Target-Disease Network}

After repetition was removed, a total of 13,523 targets related to $\mathrm{HF}$ and 15,291 targets corresponding to DM were collected from the GeneCards and the OMIM databases. In addition, 283 targets in the context of existing network analysis on actual patients with DM with HF were also obtained. Among these, 125 common targets were shared among potential targets of SGLT2 inhibitors, known HF-related targets and DM-related targets by an online Venn diagram-drawing platform, seven of which came from actual patients with DM with HF in the studies (Fig. 3), In addition, 125 "drug-disease" common targets were introduced into Cytoscape v3.6.1 software to construct a visualized drug-target-disease network (Fig. 4). A total of 131 nodes (125 targets, 4 drugs, 2 diseases) and 441 lines are shown in the network. The purple nodes are the four SGLT2 inhibitors (dapagliflozin, empagliflozin, canagliflozin and ertugliflozin); the red nodes are HF and DM; the green nodes represent 125 common targets. In this network, the average target number of each SGLT2 inhibitor is 6.73. Thus, there is an interaction between one SGLT2 inhibitor and multiple targets in the treatment of DM with $\mathrm{HF}$. With regard to the targets, the top three in degree are SLC5A2, SLC5A1, and SLC2A1, well known as the sodium-dependent glucose co-transporter gene, which can interact with the four SGLT2 inhibitors, respectively. These targets connect the relationship between SGLT2 inhibitors and disease, which provides a better reference for exploring the mechanism of SGLT2 inhibitors in the treatment of DM with HF.

\section{Core Targets of SGLT2 Inhibitors in DM Status with HF}

A total of 125 common targets were imported into the STRING online platform, and a file in "TSV" format was exported. It was then imported into Cytoscape v3.6.1, and the function "Cytohubba" was used to calculate the degree of targets to find the core targets. Thirty-three core targets of SGLT2 inhibitors in the treatment of DM with HF were screened (Fig. 5), among which red, orange, yellow and purple nodes represent a gradual decrease in the degree value from large to small. The specific information for the core targets is shown in Table 2.

\section{Analysis of 33 Core Targets of the Protein-Protein Interaction Network (PPI)}

The STRING database platform was used to construct a network of target protein interactions, and the 33 core targets of SGLT2 inhibitors-HF-DM were imported. By selecting the species "Homo sapiens" and setting a combined score $>0.9$
Fig. 2 Interaction network to indicate drug-target composited of 4 SGLT2 inhibitors (blue) and 136 targets (pink).

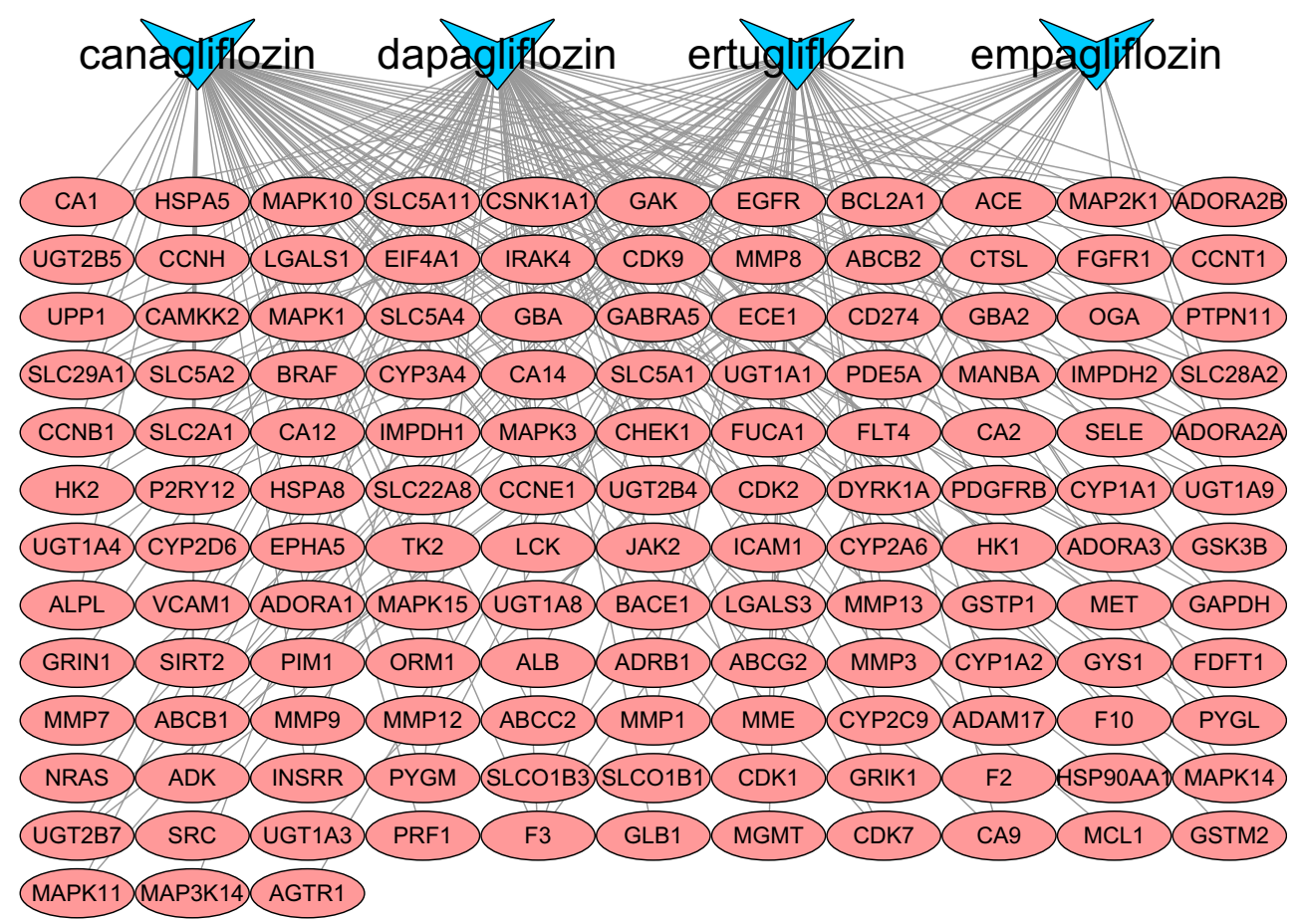


$\mathrm{HF}$

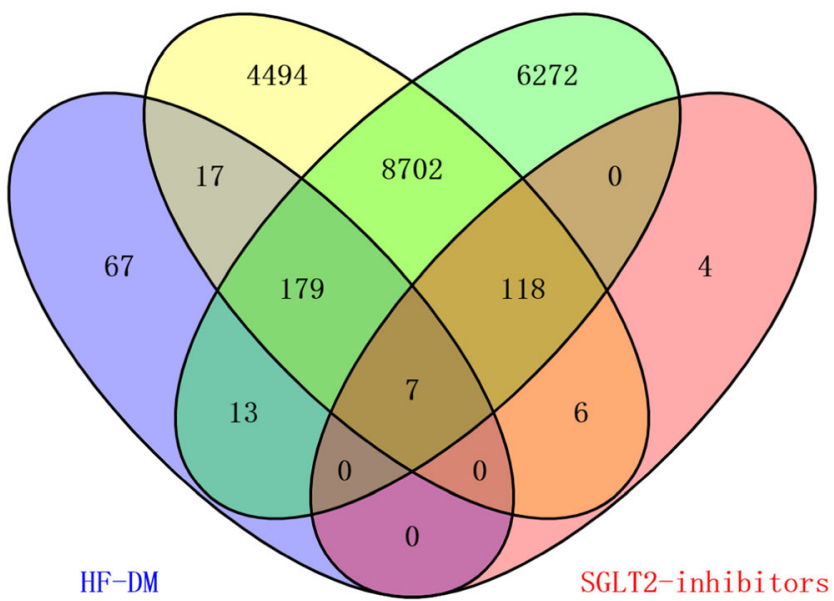

Fig. 3 The numbers of SGLT2 inhibitors and diabetes status with heart failure-related targets are shown in the Venn diagram. There were 13,523 targets of HF from the databases (upper left), 15,291 targets of DM from the database (upper right), 283 targets of HF-DM from studies (bottom left), 135 targets of SGLT2 inhibitors (bottom right) and 125 common targets between SGLT2 inhibitors and diabetes status with heart failure.

threshold, the final protein-protein interaction network was obtained (Fig. 6). As can be seen from Fig. 6, there are a total of 33 solid circles of many colors, each circle representing a key target gene, and the center of the dot shows the protein structure of the target gene. The statistical analysis was performed on each target gene to obtain the top targets with the number of adjacent genes $\geq 10$ (shown in Fig. 7), revealing that SRC, MAPK1, NARS, MAPK3 and EGFR are the top five hub targets, which may be the key targets for SGLT2 inhibitors in the treatment of DM with HF.

\section{GO Biological Function Annotation and KEGG Pathway Enrichment Analysis for Targets}

To study the mechanism of SGLT2 inhibitors in DM with HF more systematically, the 33 core target genes of the SGLT2 inhibitors-HF-DM intersection were introduced into R statistical programming language to analyze the GO biological function and KEGG signaling pathway. Filtering with $P$ value $<0.05$ and $Q$ value $<0.05$ as threshold values, we selected the 10 biological processes of 33 core targets, as shown in Fig. 8 . The results indicate that the targets are mainly associated with positive regulation of MAP kinase activity, positive regulation of protein serine/threonine kinase activity, regulation of MAP kinase activity, activation of protein kinase activity, regulation of phosphatidylinositol 3-kinase signaling, response to reactive oxygen species, phosphatidylinositol 3-kinase signaling, muscle cell proliferation, cellular response to reactive oxygen species and positive regulation of reactive oxygen species metabolic processes.
Based on the KEGG enrichment analysis of 33 key targets, we found the top 15 signal paths with high confidence (P value $<0.05$ ) selected for analysis in Fig. 9. According to the results, the core targets might affect signaling pathways including the Rap1 signaling pathway, MAPK signaling pathway, EGFR tyrosine kinase inhibitor resistance and AGERAGE signaling pathway in diabetic complications, which predicts that SGLT2 inhibitors might be effective for treatment of DM with HF by regulating the aforementioned signaling pathways.

In addition, we performed KEGG analysis on the targets of the four SGLT2 inhibitors to find their pathways separately. We intersected the pathways of the four drugs in a Venn diagram to explore the same and different signaling pathways among them (Fig. 10). We identified 387 pathways in total across four drugs. Although 33 pathways were present in a certain SGLT2 inhibitor, a total of 125 pathways were overlapped in two or more SGLT2 inhibitors, and these overlapping pathways included the four key pathways analyzed in the previous KEGG analysis results, that is, Rap1 signaling pathway, MAPK signaling pathway, EGFR tyrosine kinase inhibitor resistance and AGE-RAGE signaling pathway in diabetic complications, which demonstrates that the four pathways play an important role in the treatment of SGLT2 inhibitors in DM with HF.

\section{Discussion}

Heart failure (HF) is a major complication of diabetes mellitus (DM). Therefore, the prevention of HF in patients with DM is critical. In clinical studies, SGLT2 inhibitors were found to be effective in treating HF among patients with DM, which aroused great interest and attention. However, the mechanism is not clear. Based on network pharmacological analysis, this study explored the mechanism of SGLT2 inhibitors in the treatment of DM with HF. In the present study, four SGLT2 inhibitors (canagliflozin, dapagliflozin, empagliflozin, and ertugliflozin) were obtained to analyze the effects on DM with HF based on the network pharmacology analysis. According to the drug-disease target network and PPI network, 33 core genes of the SGLT2 inhibitors acting on DM with HF were obtained from 125 common genes. Among the 33 core genes, the top five hub targets SRC, MAPK1, NRAS, MAPK3 and EGFR were screened according to the degree. The module analysis confirmed that SGLT2 inhibitors have the potential to influence a variety of biological pathways that play an important role in the pathogenesis of DM with HF. The key pathways were screened by KEGG analysis, mainly involving the Rap1 signaling pathway, MAPK signaling pathway, EGFR tyrosine kinase inhibitor resistance and AGE-RAGE signaling pathway in diabetic complications. 


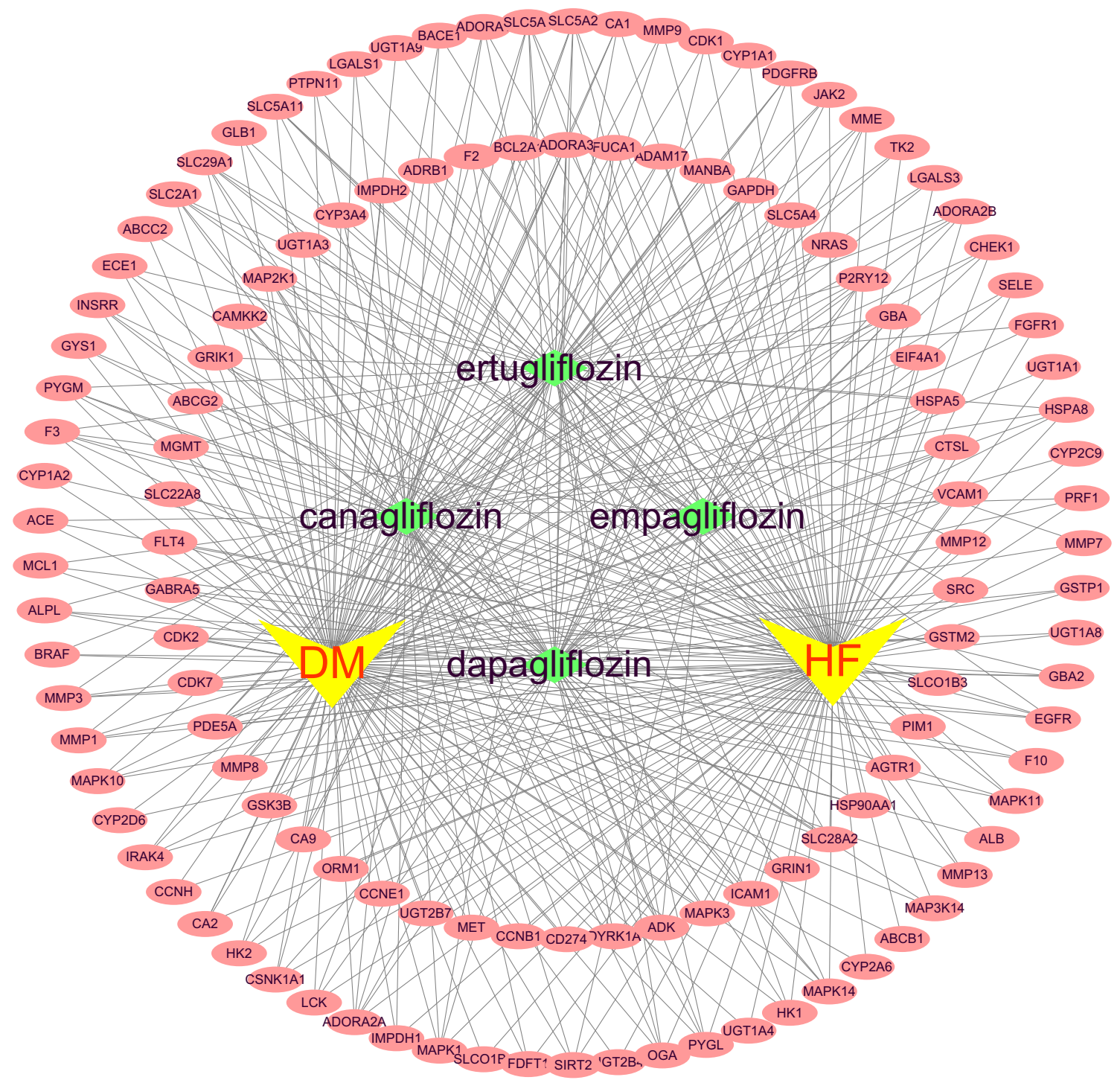

Fig. 4 The drug-target-disease networks of SGLT2 inhibitors and diabetes status with heart failure. The yellow nodes represent DM and HF; the green nodes represent four SGLT2 inhibitors; the pink nodes represent 125 common targets.

\section{Further Analysis of the Identified Genes}

Among the top five core genes, the first, SRC, is a nonreceptor tyrosine kinase that plays a role in numerous biological processes including cell adhesion, cell cycle and cell migration. It was demonstrated that SRC kinases could be activated by Ang II, which plays an important role in Ang IImediated processes [28], including the pathophysiology of cardiac hypertrophy and remodeling, vascular thickening and heart failure [29]. Pandey et al. found that SRC activation contributed to the alteration of non-myofibrillar tension, which would impact the baseline tension in fibrotic hearts after MI or in dilated cardiomyopathy [30]. Inhibition of $\mathrm{SRC}(\mathrm{c}-\mathrm{Src})$ activation was found to decrease endogenous ROS production and increase ATP production in diabetic mice with hyperlipidemia [31]. Safari-Alighiarloo et al. also identified SRC as a key gene for type 1 diabetes through analysis of gene expression profiles [32]. SRC may be a potential target for treatment of DM with HF disease.

The next two hub genes, MAPK1 (mitogen-activated protein kinase 1) and MAPK3 (mitogen-activated protein kinase 3), also known as ERK1 and ERK2, respectively, are members of the MAP kinase (MAPK) family. MAPK acts as an integration point for multiple biochemical signals and is involved in a wide variety of cellular processes including proliferation, differentiation, transcription regulation and development. As one of the MAPK family members, the activation of ERK $1 / 2$ has also been known to be involved in cardiac hypertrophy and dysfunction [33]. In addition, dysregulated ERK 1/2 levels were associated with dysregulation in a type 1 diabetes mouse model, which showed that ERK 1/2 can affect diabetes [34]. This suggests that MAPK1 and MAPK3 are potential targets in DM with HF. 


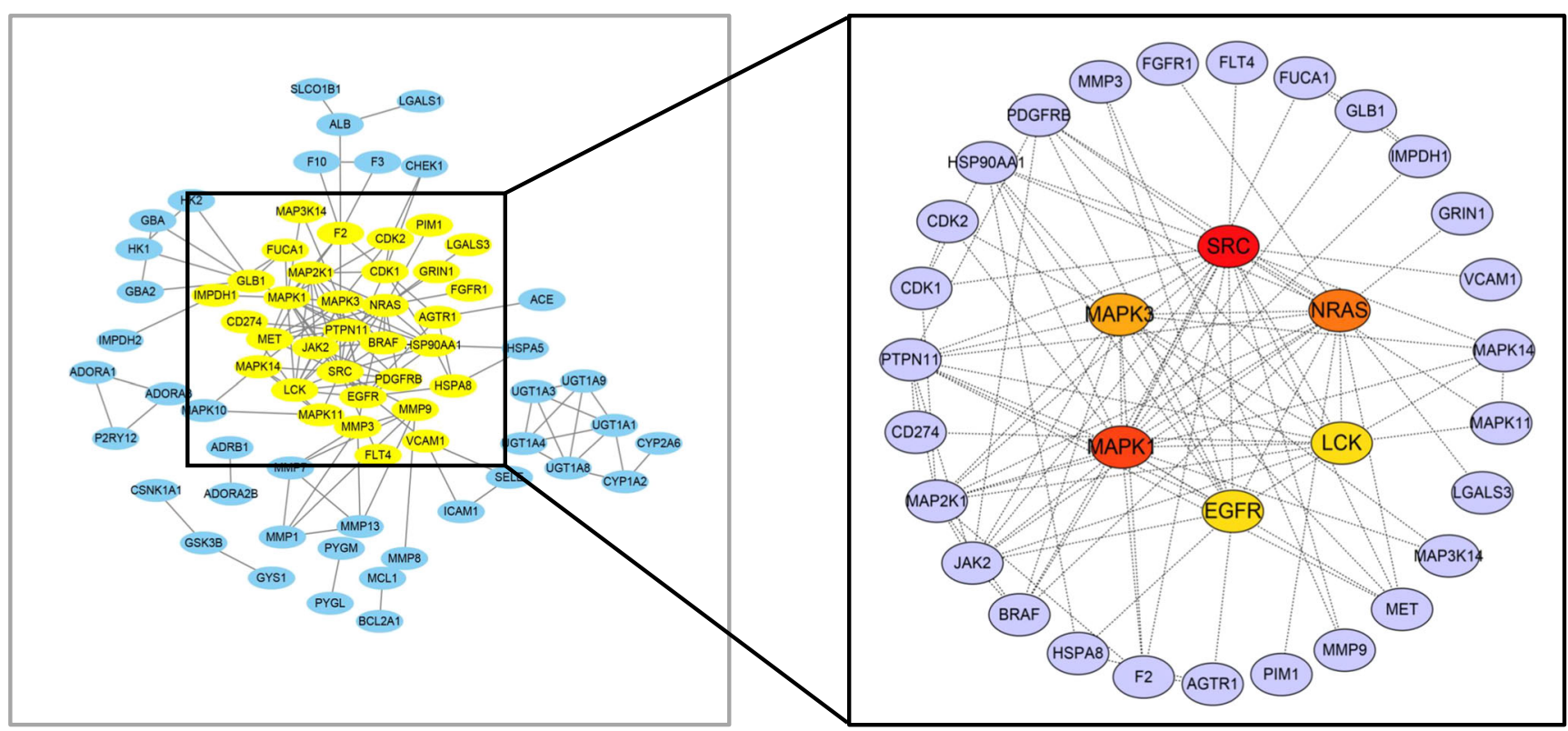

Fig. 5 The process of screening core targets by Cytohubba according to degree value. From 125 common targets (left), 33 core targets (right) ranked in the front of the degree value were selected.

The next gene is NRAS, an N-ras oncogene encoding a membrane protein that shuttles between the Golgi apparatus and the plasma membrane, and works in various differentiation processes and signal transduction involving the regulation of cell survival and growth, $\mathrm{T}$ cell activation and apoptosis. Katoh et al. reported that NRAS was one of the representative targets on cardio-miR-214 that were upregulated in human heart failure, showing that NRAS might be associated with the progression of heart failure [35]. It was also observed that hispidulin modulated multiple Pim1-interacted proteins such as NRAS and RAB18 to regulate the development of diabetic nephropathy [36]. Thus, NRAS has the potential to be a target of DM with HF.

The last of the top five hub genes is EGFR, epidermal growth factor receptor, a cell surface protein binding to epidermal growth factor that regulates cell growth, proliferation and survival, and is involved in blood pressure regulation, neointimal hyperplasia, atherogenesis and reactive oxygen. $\mathrm{Xu}$ et al. revealed that the enhanced myogenic constriction of the mesenteric artery in heart failure might be related to the loss of plasmalemmal caveolae in mesenteric vascular smooth muscle cells, and the increased activity of the EGFR and $\mathrm{AT}_{1}$ receptors was considered to be one of the mechanisms leading to this result [37]. Belmadani et al. found that elevated EGFR phosphorylation contributed to resistance artery dysfunction in type 2 diabetes [38]. Zhang et al. concluded that inhibiting EGFR slowed the progression of diabetic nephropathy by decreasing endoplasmic reticulum stress and increasing autophagy [39]. These findings demonstrate that EGFR may play an irreplaceable role in DM with HF. Overall, the top five core genes of the present study based on network pharmacology are supported by previous studies.
Moreover, the targets of our findings have some overlap with those in existing network analysis on actual patients with DM with HF, that is ALB, FDFT1, SLC22A8, ABCG2, MME, LGALS3 and FUCA1, which act as biomarkers in HF patients with DM $[26,27]$. The results suggest that these targets may play an important role in the prevention and treatment of SGLT2 inhibitors in DM combined with heart failure.

\section{Enrichment Analysis Based on Core Targets}

GO functional enrichment and KEGG pathway analysis have illustrated the role of the SGLT2 inhibitors in the gene function and signaling pathway. According to the biological processes that mainly reflect the GO functional enrichment, these core targets are focused on kinase activity, primarily in positive regulation of MAP kinase activity, positive regulation of protein serine/threonine kinase activity and so on. Among them, positive regulation of protein serine/threonine kinase activity is a core biological process that may affect the function of cGMP in the regulation of the physiological response of the natriuretic peptide (NP) system. This is because cGMPdependent protein kinases (cGK) are exactly serine/threonine kinases widely distributed in eukaryotes [40]. The physiological response of the NP system is mainly achieved by binding to NPR-A, activating guanosine cyclase and producing cGMP [41], and the NP system has been extensively associated with the development and progression of HF [42]. It seems that SGLT2 inhibitors can affect the NP system to act on the development of HF through the biological process involving positive regulation of protein serine/threonine kinase activity.

In the enrichment of the KEGG pathway, the Rap1 signaling pathway, MAPK signaling pathway, EGFR tyrosine kinase 
Table 2 Specific information for core targets

\begin{tabular}{|c|c|c|c|c|}
\hline Number & Uniprot ID & Gene name & Protein name & Counts \\
\hline 1 & P12931 & SRC & Tyrosine-protein kinase Lck & 19 \\
\hline 2 & P28482 & MAPK1 & Mitogen-activated protein kinase 1 & 17 \\
\hline 3 & P01111 & NRAS & GTPase NRas & 16 \\
\hline 4 & P27361 & MAPK3 & Mitogen-activated protein kinase 3 & 15 \\
\hline 5 & P00533 & EGFR & Epidermal growth factor receptor & 12 \\
\hline 6 & P06239 & LCK & Tyrosine-protein kinase Lck & 12 \\
\hline 7 & Q06124 & PTPN11 & $\begin{array}{l}\text { Tyrosine-protein phosphatase nonreceptor } \\
\text { type } 11\end{array}$ & 11 \\
\hline 8 & Q02750 & MAP2K1 & $\begin{array}{l}\text { Dual specificity mitogen-activated } \\
\text { protein kinase kinase } 1\end{array}$ & 11 \\
\hline 9 & O60674 & JAK2 & Tyrosine-protein kinase JAK2 & 10 \\
\hline 10 & P07900 & HSP90AA1 & Heat shock protein HSP 90 -alpha & 7 \\
\hline 11 & P09619 & PDGFRB & $\begin{array}{l}\text { Platelet-derived growth factor receptor } \\
\text { beta }\end{array}$ & 6 \\
\hline 12 & P15056 & BRAF & Serine/threonine-protein kinase B-raf & 6 \\
\hline 13 & Q16539 & MAPK14 & Mitogen-activated protein kinase 14 & 5 \\
\hline 14 & P00734 & $\mathrm{F} 2$ & Prothrombin & 5 \\
\hline 15 & P08581 & MET & Hepatocyte growth factor receptor & 5 \\
\hline 16 & P06493 & CDK1 & Cyclin-dependent kinase 1 & 4 \\
\hline 17 & P24941 & $\mathrm{CDK} 2$ & Cyclin-dependent kinase 2 & 3 \\
\hline 18 & P30556 & AGTR1 & Type-1 angiotensin II receptor & 3 \\
\hline 19 & P11142 & HSPA8 & Heat shock cognate $71 \mathrm{kDa}$ protein & 3 \\
\hline 20 & Q15759 & MAPK11 & Mitogen-activated protein kinase 11 & 3 \\
\hline 21 & P04066 & FUCA1 & Tissue alpha-L-fucosidase & 3 \\
\hline 22 & P16278 & GLB1 & Beta-galactosidase & 3 \\
\hline 23 & P20839 & IMPDH1 & Inosine-5'-monophosphate dehydrogenase 1 & 3 \\
\hline 24 & Q9NZQ7 & $\mathrm{CD} 274$ & Programmed cell death 1 ligand 1 & 2 \\
\hline 25 & P14780 & MMP9 & Matrix metalloproteinase-9 & 2 \\
\hline 26 & P08254 & MMP3 & Stromelysin-1 & 2 \\
\hline 27 & Q99558 & MAP3K14 & Mitogen-activated protein kinase kinase kinase 14 & 2 \\
\hline 28 & Q05586 & GRIN1 & Glutamate receptor ionotropic, NMDA 1 & 1 \\
\hline 29 & P19320 & VCAM1 & Vascular cell adhesion protein 1 & 1 \\
\hline 30 & P11309 & PIM1 & Serine/threonine-protein kinase pim-1 & 1 \\
\hline 31 & P17931 & LGALS3 & Galectin-3 & 1 \\
\hline 32 & P11362 & FGFR1 & Fibroblast growth factor receptor 1 & 1 \\
\hline 33 & P35916 & FLT4 & Vascular endothelial growth factor receptor 3 & 1 \\
\hline
\end{tabular}

inhibitor resistance and AGE-RAGE signaling pathway in diabetic complications were found to be the significant pathways.

The Rap1 signaling pathway is implicated in a wide range of biological processes from cell proliferation and differentiation to cell adhesion. Rap1 has been proven to play a part in the regulation of integrin affinity, adhesion, and migration in postnatal neovascularization, mainly mediating the angiogenesis pathway and serving in an important role in cardiac hypertrophy. Furthermore, Rap1B can prevent excessive vascular leakage in early diabetes mellitus by inhibiting VEGF signal transduction. By controlling telomere length, Rap1 can decrease the occurrence and development of diabetes-related cardiovascular disease
[43]. Downregulation of Rap1B to reduce VEGF signal transduction can impede excessive vascular leakage in early diabetes mellitus [44]. These imply that the Rap1 signaling pathway may be involved in DM with HF.

Consistent with the core target results above, the MAPK pathway is also predicted to play a role in DM with HF. It was demonstrated that the MAPK signaling pathway cascade is initiated in cardiomyocytes through activation of $G$ proteincoupled receptors, receptor tyrosine kinases and stress stimulation. Zhang et al. reported that the MAPK signaling pathway was found to regulate cardiomyocyte apoptosis in mice with heart failure after MI, indicating that this pathway has a 
Fig. 6 The protein-protein interaction (PPI) network based on 33 core targets of SGLT2 inhibitors in diabetes status with heart failure. Network nodes represent different proteins. The structures in the nodes are the protein structures. Edges represent proteinprotein associations, and the line thickness indicates the strength of data support.

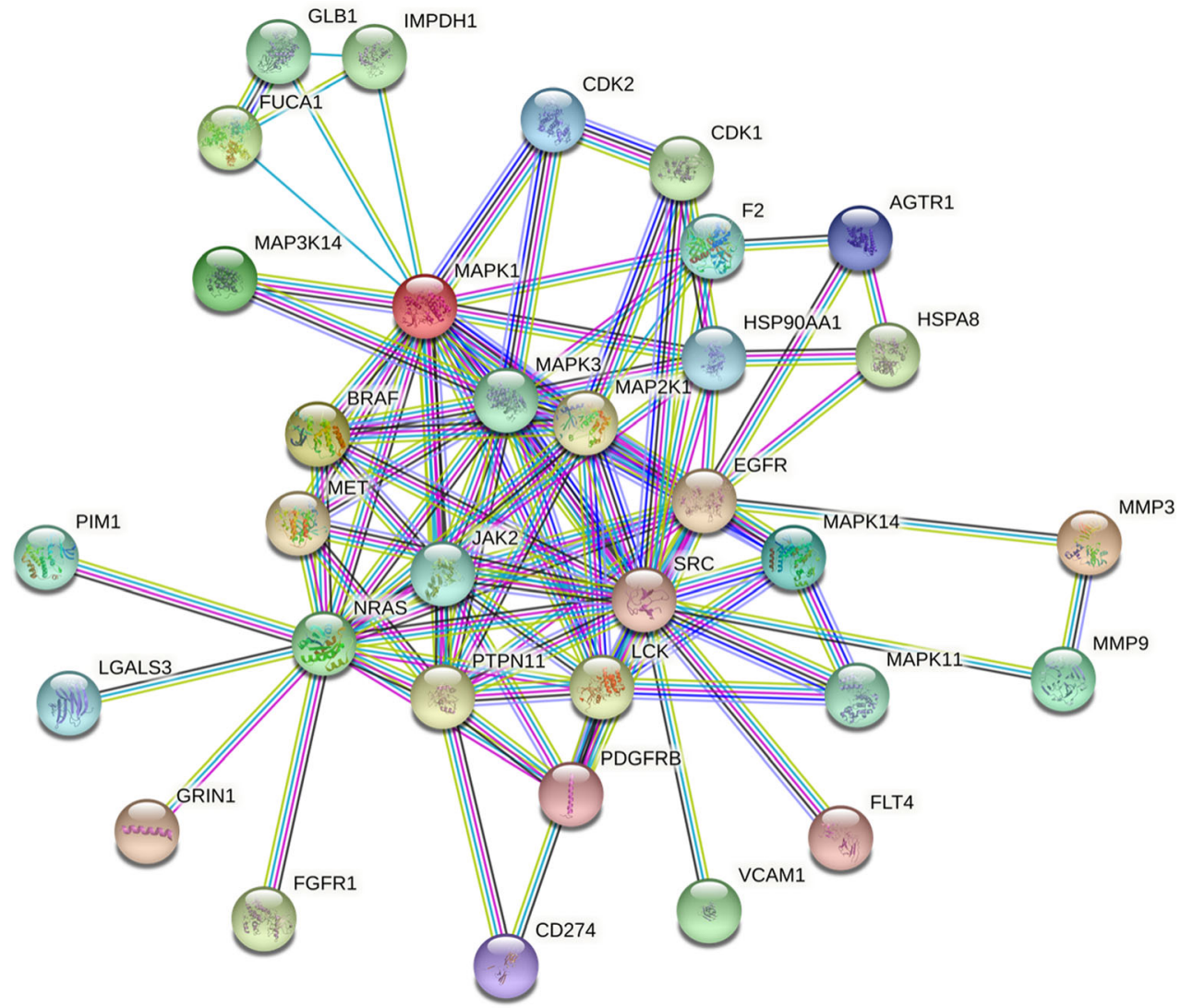

potential role in heart failure disease [45]. During the process of insulin resistance, there exists a chronic inflammatory response involving the MAPK pathway in the inflammatory response of type 2 diabetes, such as in diabetic nephropathy and liver disease [46, 47].

Fig. 7 Protein interaction relationship histogram of SGLT2 inhibitors.

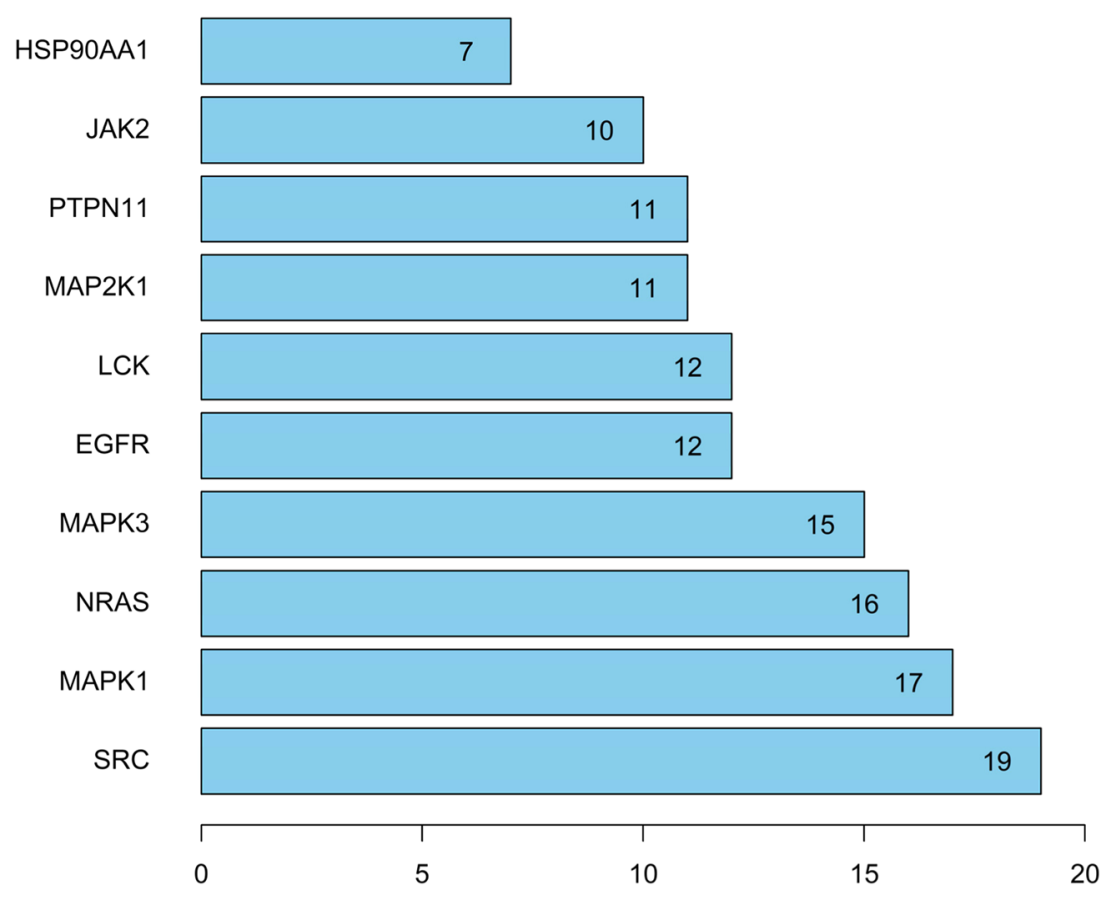


Fig. 8 Enrichment analysis of Gene Ontology (GO) biological process of 33 core genes related to SGLT2 inhibitors on diabetes status with heart failure.

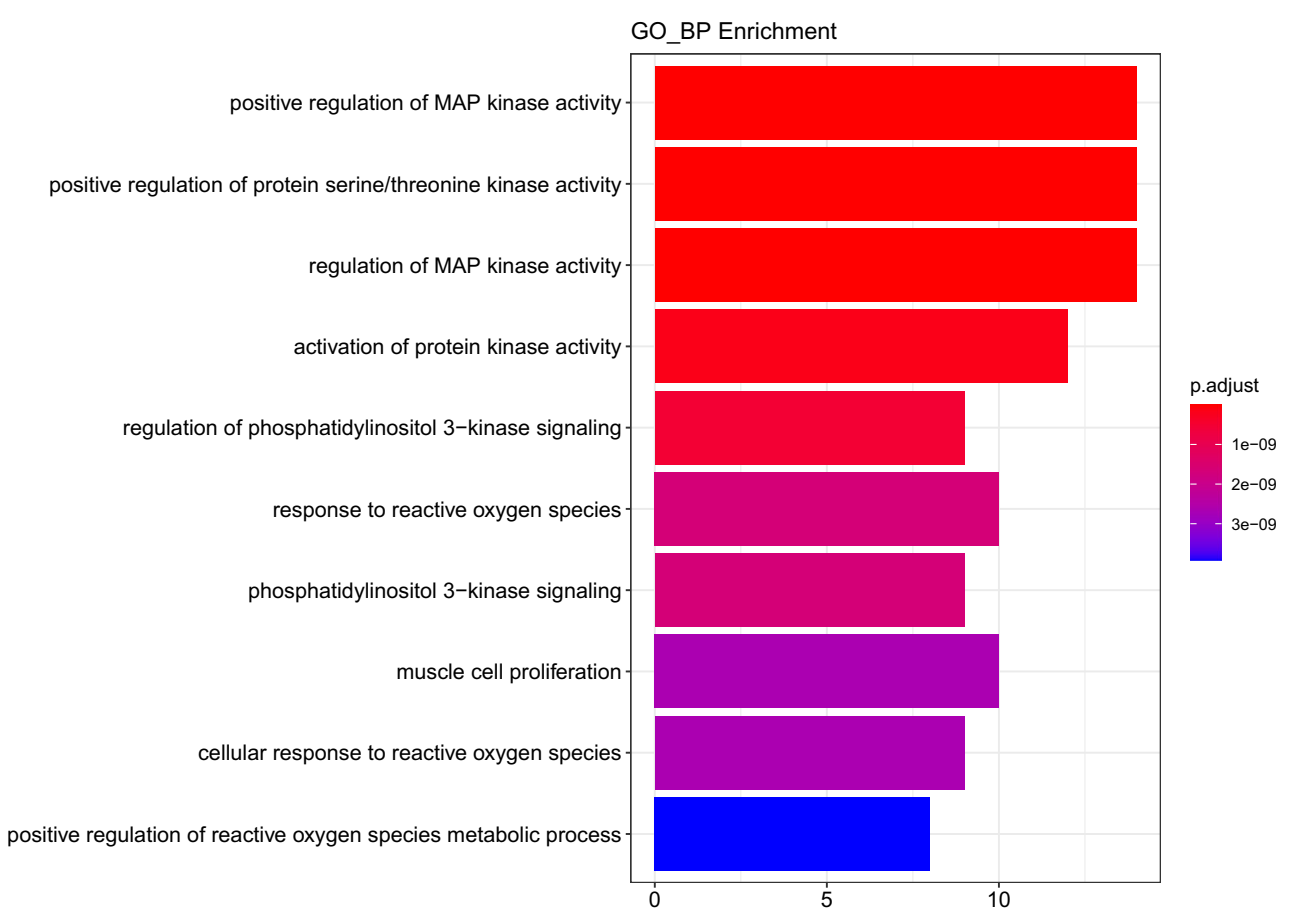

inhibition reduced ROS production in the left ventricle and blunted hypertensive myocardial hypertrophy in spontaneously hypertensive rats [49]. Therefore, the EGFR tyrosine kinase inhibitor resistance may have a common effect to accelerate the progression of DM with HF.

The AGE-RAGE signaling pathway is a well-studied cascade in DM. It is found that the AGE-RAGE signaling pathway can directly mediate vascular calcification in diabetes. Additionally, the pathway can also impact diabetic complications, as it leads to oxidative stress, increased inflammation, and enhanced extracellular matrix accumulation resulting in diastolic and systolic dysfunction [50]. Fukami et al. proved that activation of the AGERAGE signaling pathway in diabetic complications could cause excessive production of advanced glycation end products to damage cardiomyocytes, leading to HF [51], which indicates that the AGE-RAGE signaling pathway in diabetic complications might mediate the progression of heart failure, similar to results of pathway analysis in another study [52]. Therefore, our results
Fig. 9 Kyoto Encyclopedia of Genes and Genomes (KEGG) pathway for 33 core targets of SGLT2 inhibitors on diabetes status with heart failure.

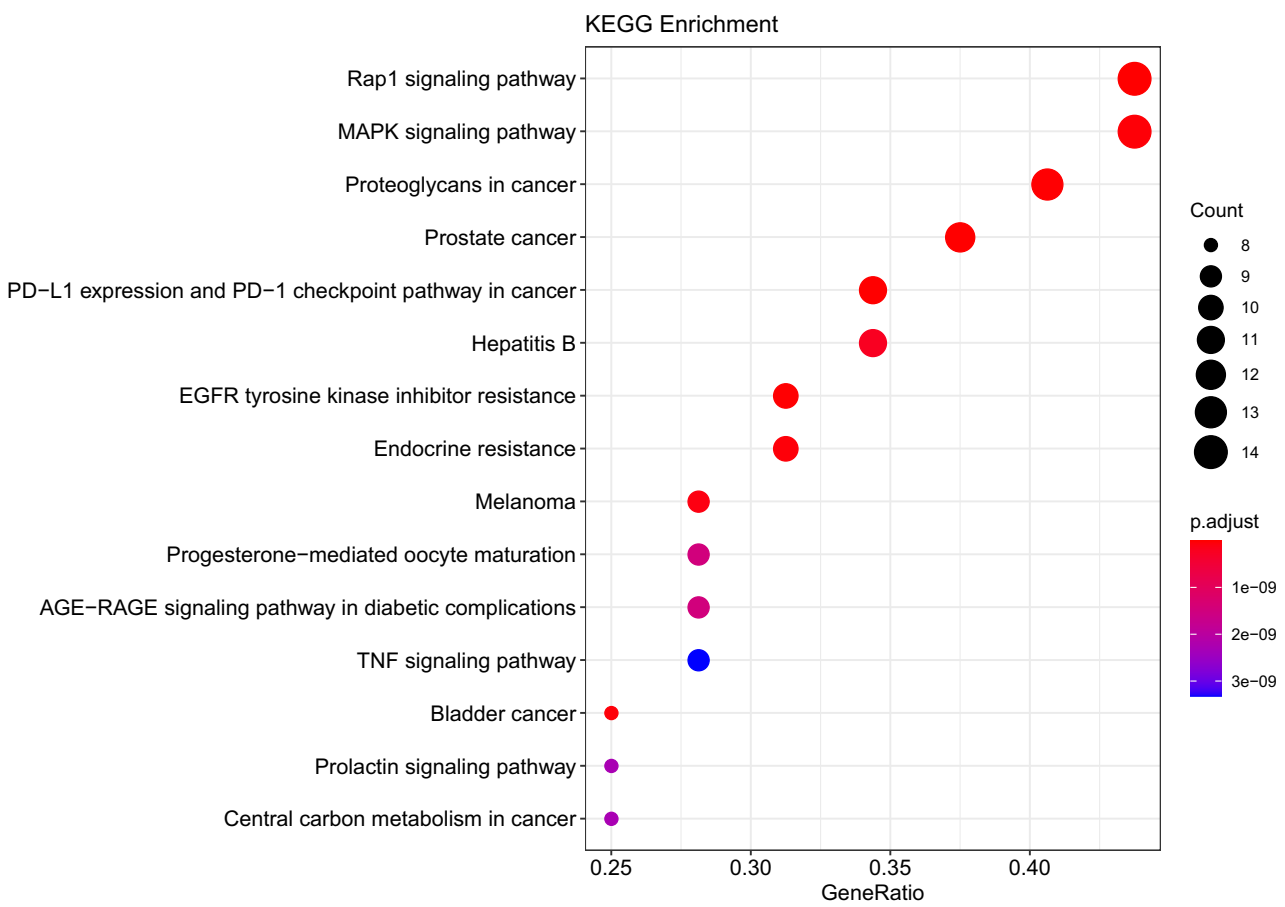




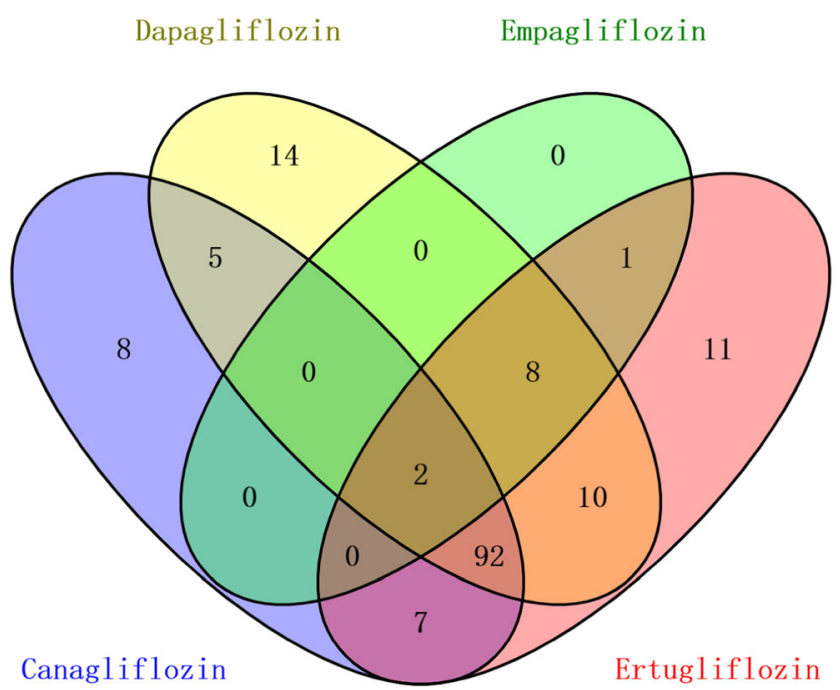

Fig. 10 The KEGG pathways of the four drugs were shown in the Venn diagram.

suggest that the four signaling pathways may be involved in the mechanisms of SGLT2 inhibitors affecting DM status with HF.

Meanwhile, other KEGG analysis results that explore the same and different signaling pathways between four SGLT2 inhibitors showed that the four key pathways mentioned above were contained in 125 pathways overlapping in two or more SGLT2 inhibitors, which also indicated that the four pathways might play a key part in the treatment of DM with HF by SGLT2 inhibitors. In addition, according to the same and different mechanistic pathways of four SGLT2 inhibitors, we suggest that even though the four SGLT2 inhibitors have a few different pathways, a large part of their pathways of action are similar. The four SGLT2 inhibitors have a common parent nucleus, although their side-chain substituents are somewhat different, leading to small differences in the efficacy of drugs [13]. Considering these factors, we think that the four SGLT2 inhibitors may have class effects on the whole, but do not rule out that they each have unique effects in some diseases. Some studies have also indicated that SGLT2 inhibitors had more internally and externally consistent class effects on HF risk reduction than the MACE composite outcome [53, 54].

Network pharmacology is indeed a new method for studying the relationship between drugs and diseases. In our study, network pharmacology revealed the potential targets of SGLT2 inhibitors, as well as targets of DM with HF, and bioinformatics was used to study the main enrichment pathways. Based on the network pharmacology, our study predicted the potential therapeutic targets of SGLT2 inhibitors in DM with HF and revealed their action on the main pathways through core genes, which explained the mechanisms of SGLT2 inhibitors in DM with HF and provided scientific evidence for SGLT2 inhibitors to treat DM with HF. However, the main limitation of this study is the lack of experimental verification. Consequently, pharmacological studies will be critical to further elucidate the relationship of SGLT2 inhibitors and DM with HF. In addition, validation of the molecular levels of our findings is necessary for the future.

\section{Conclusions}

Taken together, our study systematically predicted, screened and analyzed the targets and pathways that might play a vital role in the biological process, which elaborated the possible mechanisms of SGLT2 inhibitors in DM status with HF. Most importantly, these results provide evidence and new insights for further research on the pharmacological mechanism of SGLT2 inhibitors.

Supplementary Information The online version contains supplementary material available at https://doi.org/10.1007/s10557-021-07186-y.

Author Contributions (I) Conception and design: Ziling Mai, Huanqiang Li, Yong Liu, Jiyan Chen; (II) Administrative support: Enzhao Chen, Shiqun Chen; (III) Collection and assembly of data: Zhubin Lun, Huanqiang Li, Liwei Liu, Wenguang Lai; (IV) Data analysis and interpretation: Ziling Mai, Guanzhong Chen, Enzhao Chen; (V) Manuscript writing: All authors; (VI) Final approval of manuscript: All authors.

Funding This study was supported by the National Science Foundation of China (Grant No. 81670339 and Grant No. 81970311), Cardiovascular Research Foundation Project of the Chinese Medical Doctor Association (SCRFCMDA201216), the Progress of Science and Technology Project in Guangdong Province (2017B020247060), Beijing Lisheng Cardiovascular Health Foundation (LHJJ20141751 and LHJJ201612127), Natural Science Foundation of Guangdong Province General Project (2020A1515010940), High-level talent team building project (Y012018085), Guangdong Provincial Fund for Clinical Medications (2019ZH01) and Dengfeng Project in Guangdong Province (DFJH201919 and DFJH2020026). The funding body plays no direct role in the design of the study, and collection, analysis, and interpretation of data, and in writing the manuscript.

Data Availability Author can confirm that all relevant data are included in the article and/or its supplementary information files.

\section{Declarations}

Ethical Approval This article does not contain any studies with human participants or animals performed by any of the authors.

Informed Consent This article does not contain any studies with human participants performed by any of the authors.

Consent for publication All authors agree to public.

Conflict of Interest The authors declare that there is no conflict of interest.

Open Access This article is licensed under a Creative Commons Attribution 4.0 International License, which permits use, sharing, adaptation, distribution and reproduction in any medium or format, as 
long as you give appropriate credit to the original author(s) and the source, provide a link to the Creative Commons licence, and indicate if changes were made. The images or other third party material in this article are included in the article's Creative Commons licence, unless indicated otherwise in a credit line to the material. If material is not included in the article's Creative Commons licence and your intended use is not permitted by statutory regulation or exceeds the permitted use, you will need to obtain permission directly from the copyright holder. To view a copy of this licence, visit http://creativecommons.org/licenses/by/4.0/.

\section{References}

1. World Health Organization (WHO) (2016) Global Report on Diabetes.; Available from: http://apps.who.int/h/iris/bitstream/ 10665/204871/1/9789241565257_eng.pdf.

2. Saeedi P, et al. Global and regional diabetes prevalence estimates for 2019 and projections for 2030 and 2045: Results from the International Diabetes Federation Diabetes Atlas, 9(th) edition. Diabetes Res Clin Pract. 2019;157107843.

3. Kenny HC, Abel ED. Heart Failure in Type 2 Diabetes Mellitus. Circ Res. 2019;124(1):121-41.

4. Avogaro A, et al. Incidence of heart failure in patients with type 1 diabetes: a systematic review of observational studies. J Endocrinol Invest. 2020.

5. Palau P, et al. Differential prognostic impact of type 2 diabetes mellitus in women and men with heart failure with preserved ejection fraction. Rev Esp Cardiol (Engl Ed). 2020;73(6):463-70.

6. Rawshani A, et al. Risk Factors, Mortality, and Cardiovascular Outcomes in Patients with Type 2 Diabetes. N Engl J Med. 2018;379(7):633-44.

7. Ziaeian B, et al. Long-term outcomes for heart failure patients with and without diabetes: From the Get With The Guidelines-Heart Failure Registry. Am Heart J. 2019;211:1-10.

8. Singh JS, et al. Research into the effect Of SGLT2 inhibition on left ventricular remodelling in patients with heart failure and diabetes mellitus (REFORM) trial rationale and design. Cardiovasc Diabetol. 2016;15:97.

9. Hippisley-Cox J, Coupland C. Diabetes treatments and risk of heart failure, cardiovascular disease, and all cause mortality: cohort study in primary care. Bmj. 2016;354:i3477.

10. Kunavisarut T, Sriussadaporn S, Lertwattanarak R. Beta-cell function in type 2 diabetic patients who failed to maintain good glycemic status with a combination of maximum dosages of metformin and sulfonylurea. Diabetes Metab Syndr Obes. 2019;12:761-70.

11. Seferović PM, et al. Type 2 diabetes mellitus and heart failure: a position statement from the Heart Failure Association of the European Society of Cardiology. Eur J Heart Fail. 2018;20(5): 853-72.

12. Vaduganathan M, Januzzi JL Jr. Preventing and Treating Heart Failure with Sodium-Glucose Co-Transporter 2 Inhibitors. Am J Cardiol. 2019;124(Suppl 1):S20-s27.

13. Rieg T, Vallon V. Development of SGLT1 and SGLT2 inhibitors. Diabetologia. 2018;61(10):2079-86.

14. Udell JA, et al. Cardiovascular Outcomes and Risks After Initiation of a Sodium Glucose Cotransporter 2 Inhibitor: Results From the EASEL Population-Based Cohort Study (Evidence for Cardiovascular Outcomes With Sodium Glucose Cotransporter 2 Inhibitors in the Real World). Circulation. 2018;137(14):1450-9.

15. Zinman B, et al. Empagliflozin, Cardiovascular Outcomes, and Mortality in Type 2 Diabetes. N Engl J Med. 2015;373(22):211728.
16. Kosiborod M, et al. Cardiovascular Events Associated With SGLT2 Inhibitors Versus Other Glucose-Lowering Drugs: The CVDREAL 2 Study. J Am Coll Cardiol. 2018;71(23):2628-39.

17. Fadini GP, et al. Effectiveness of dapagliflozin versus comparators on renal endpoints in the real world: A multicentre retrospective study. Diabetes Obes Metab. 2019;21(2):252-60.

18. Pasternak B, et al. Use of sodium-glucose co-transporter 2 inhibitors and risk of serious renal events: Scandinavian cohort study. Bmj. 2020;369:m1186.

19. Neal B, et al. Canagliflozin and Cardiovascular and Renal Events in Type 2 Diabetes. N Engl J Med. 2017;377(7):644-57.

20. Wiviott SD, et al. Dapagliflozin and Cardiovascular Outcomes in Type 2 Diabetes. N Engl J Med. 2019;380(4):347-57.

21. Cosentino F, et al. Efficacy of Ertugliflozin on Heart FailureRelated Events in Patients With Type 2 Diabetes Mellitus and Established Atherosclerotic Cardiovascular Disease: Results of the VERTIS CV Trial. Circulation. 2020;142(23):2205-15.

22. McMurray JJV, et al. Dapagliflozin in Patients with Heart Failure and Reduced Ejection Fraction. N Engl J Med. 2019;381(21): 1995-2008.

23. Ning K, et al. Computational Molecular Networks and Network Pharmacology. Biomed Res Int. 2017;2017:7573904.

24. Daina A, Michielin O, Zoete V. SwissTargetPrediction: updated data and new features for efficient prediction of protein targets of small molecules. Nucleic Acids Res. 2019;47(W1):W357-w364.

25. Wishart DS, et al. HMDB 4.0: the human metabolome database for 2018. Nucleic Acids Res. 2018;46(D1):D608-d617.

26. Sharma A, et al. A network analysis to compare biomarker profiles in patients with and without diabetes mellitus in acute heart failure. Eur J Heart Fail. 2017;19(10):1310-20.

27. Tromp J, et al. Distinct Pathological Pathways in Patients With Heart Failure and Diabetes. JACC Heart Fail. 2020;8(3):234-42.

28. Bali A, Jaggi AS. Angiotensin II-triggered kinase signaling cascade in the central nervous system. Rev Neurosci. 2016;27(3):301-15.

29. Peng K, et al. Novel EGFR inhibitors attenuate cardiac hypertrophy induced by angiotensin II. J Cell Mol Med. 2016;20(3):482-94.

30. Pandey P, et al. Cardiomyocytes Sense Matrix Rigidity through a Combination of Muscle and Non-muscle Myosin Contractions. Dev Cell. 2018;45(5):661.

31. Sato $\mathrm{Y}$, et al. Palmitate induces reactive oxygen species production and $\beta$-cell dysfunction by activating nicotinamide adenine dinucleotide phosphate oxidase through Src signaling. J Diabetes Investig. 2014;5(1):19-26.

32. Safari-Alighiarloo N, et al. Identification of new key genes for type 1 diabetes through construction and analysis of protein-protein interaction networks based on blood and pancreatic islet transcriptomes. J Diabetes. 2017;9(8):764-77.

33. $\mathrm{Xu} \mathrm{Z}$, et al. The Role of ERK1/2 in the Development of Diabetic Cardiomyopathy. Int J Mol Sci. 2016:17(12).

34. Galvão Tessaro FH, et al. Macrophages from a type 1 diabetes mouse model present dysregulated P13K/AKT, ERK $1 / 2$ and SAPK/JNK levels. Immunobiology. 2020;225(2):151879.

35. Katoh M. Cardio-miRNAs and onco-miRNAs: circulating miRNAbased diagnostics for non-cancerous and cancerous diseases. Front Cell Dev Biol. 2014;2:61.

36. $\mathrm{Wu} \mathrm{F}$, et al. Hispidulin alleviates high-glucose-induced podocyte injury by regulating protective autophagy. Biomed Pharmacother. 2018;104:307-14.

37. $\mathrm{Xu} \mathrm{Y,} \mathrm{et} \mathrm{al.} \mathrm{Enhanced} \mathrm{myogenic} \mathrm{constriction} \mathrm{of} \mathrm{mesenteric} \mathrm{artery} \mathrm{in}$ heart failure relates to decreased smooth muscle cell caveolae numbers and altered AT1- and epidermal growth factor-receptor function. Eur J Heart Fail. 2009;11(3):246-55.

38. Belmadani S, et al. Elevated epidermal growth factor receptor phosphorylation induces resistance artery dysfunction in diabetic $\mathrm{db} / \mathrm{db}$ mice. Diabetes. 2008;57(6):1629-37. 
39. Zhang MZ, et al. Epidermal growth factor receptor inhibition slows progression of diabetic nephropathy in association with a decrease in endoplasmic reticulum stress and an increase in autophagy. Diabetes. 2014;63(6):2063-72.

40. Hofmann F, Wegener JW. cGMP-dependent protein kinases (cGK). Methods Mol Biol. 2013;1020:17-50.

41. Volpe M, Carnovali M, Mastromarino V. The natriuretic peptides system in the pathophysiology of heart failure: from molecular basis to treatment. Clin Sci (Lond). 2016;130(2):57-77.

42. Abuzaanona A, Lanfear D. Pharmacogenomics of the Natriuretic Peptide System in Heart Failure. Curr Heart Fail Rep. 2017;14(6): 536-42.

43. Cai Y, et al. Decoding telomere protein Rap1: Its telomeric and nontelomeric functions and potential implications in diabetic cardiomyopathy. Cell Cycle. 2017;16(19):1765-73.

44. Lakshmikanthan $\mathrm{S}$, et al. Rap1B promotes VEGF-induced endothelial permeability and is required for dynamic regulation of the endothelial barrier, J Cell Sci. 2018;131(1).

45. Zhang Q, et al. MAPK pathway regulated the cardiomyocyte apoptosis in mice with post-infarction heart failure. Bratisl Lek Listy. 2017;118(6):339-46.

46. Bak EJ, et al. Licochalcone F alleviates glucose tolerance and chronic inflammation in diet-induced obese mice through Akt and p38 MAPK. Clin Nutr. 2016;35(2):414-21.

47. Zhao Y, et al. TAB3 involves in hepatic insulin resistance through activation of MAPK pathway. Gen Comp Endocrinol. 2015;224: 228-34.
48. Li Z, et al. Inhibition of Epidermal Growth Factor Receptor Activation Is Associated With Improved Diabetic Nephropathy and Insulin Resistance in Type 2 Diabetes. Diabetes. 2018;67(9): 1847-57.

49. Zeng SY, et al. Inhibition of the ROS-EGFR Pathway Mediates the Protective Action of Nox1/4 Inhibitor GKT137831 against Hypertensive Cardiac Hypertrophy via Suppressing Cardiac Inflammation and Activation of Akt and ERK1/2. Mediators Inflamm. 2020;2020:1078365.

50. Bodiga VL, Eda SR, Bodiga S. Advanced glycation end products: role in pathology of diabetic cardiomyopathy. Heart Fail Rev. 2014;19(1):49-63.

51. Fukami K, Yamagishi S, Okuda S. Role of AGEs-RAGE system in cardiovascular disease. Curr Pharm Des. 2014;20(14):2395-402.

52. Tao YG, et al. Exploring Molecular Mechanism of Huangqi in Treating Heart Failure Using Network Pharmacology. Evid Based Complement Alternat Med. 2020;2020:6473745.

53. Giugliano D, Meier JJ, Esposito K. Heart failure and type 2 diabetes: From cardiovascular outcome trials, with hope. Diabetes Obes Metab. 2019;21(5):1081-7.

54. Kluger AY, et al. Class effects of SGLT2 inhibitors on cardiorenal outcomes. Cardiovasc Diabetol. 2019;18(1):99.

Publisher's Note Springer Nature remains neutral with regard to jurisdictional claims in published maps and institutional affiliations. 